\title{
Diode-pumped, high-energy, single transverse mode a-switch fibre laser
}

1662

\author{
D.J. Richardson, P. Britton and D. Taverner
}

The authors report the generation of $180 \mu \mathrm{J}, 3.6 \mathrm{~kW}$ peak power pulses from a simple, diode-pumped large mode erbium doped fibre Q-switch laser system. This is believed to be the highest energy ever obtained from a single transverse mode fibre laser system.

Actively Q-switched, erbium-doped fibre lasers offer a simple and robust means for the generation of high energy, nanosecond pulses at eye-safe wavelengths around $1550 \mathrm{~nm}$ suitable for a number of industrial, sensing and nonlinear optics applications [1 - 4]. For many of these applications, a high-quality, spatial mode-profile is a fundamental requirement, making single transverse mode per-formance a necessity. The maximum pulse energy we can obtain from a Q-switch system is ultimately determined by the energy storage characteristics of the gain medium. Conventional commer- cial erbium doped fibres are optimised for amplifier applications where a high gain efficiency is the main design criteria. Unfortu-nately, this optimisation, which demands the use of a tightly con-fined mode, compromises the energy storage characteristics of the fibre, since energy is rapidly lost in the form of amplified sponta-neous emission (ASE) as the pump power to the system is increased. If we use conventional erbium doped fibre, the maxi-mum extractable energy in a single Q-switch pulse is limited to $\sim 10 \mu \mathrm{J}$. Recently, we have experimentally demonstrated that the use of a large mode area, low numerical aperture, singlemode waveguide design significantly reduces the small signal gain eff-ciency and thereby greatly increases the energy storage capacity of the fibre $[5,6]$. Using such an approach, we achieved record actively Q-switched fibre laser pulse energies of $50 \mu \mathrm{J}$ at pump powers levels $(-600 \mathrm{~m} \mathrm{~W})$ commensurate with semiconductor laser diode pumping [7]. In this Letter, we present our latest results based on this concept. Using a laser diode pump source and a fibre with further increased mode area, we have improved the laser performance, obtaining pulse energies as high as $180 \mu$ with corre-sponding pulse powers in excess of $3 \mathrm{~kW}$. To the best of our knowledge, this is the highest pulse energy ever reported for a single transverse mode fibre system.

Fig. 1 Laser configuration

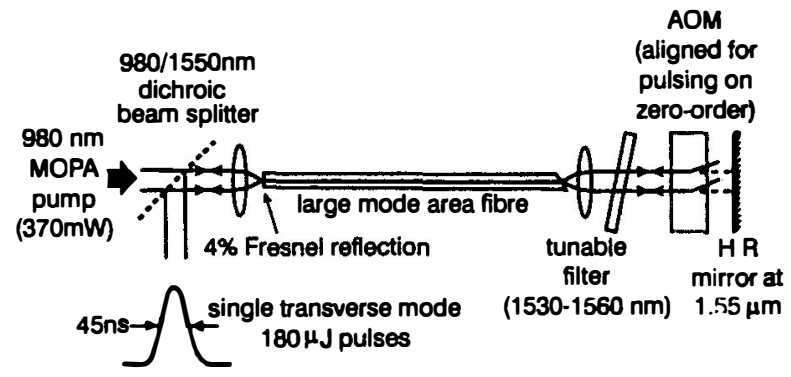

The laser cavity is shown in Fig. 1. Pump light from an isolated 980nm M0PA semiconductor pump source delivering $370 \mathrm{~mW}$ is launched into the large mode area fibre through a $980 / 1550 \mathrm{~nm}$ dichroic beam splitter using an appropriately matched lens combination. We estimate a launch efficiency of $80 \%$. The $4 \mathrm{~m}$ long fibre. doped with $400 \mathrm{ppm}$ of $\mathrm{Er}^{3-}$ ions, had an NA of 0.066 , and a cutoff wavelength of $1450 \mathrm{~nm}$ and was therefore single transverse mode at the operating wavelength. The estimated mode-field area for this fibre was $310 \mu \mathrm{m}^{2}$ as compared to the $30-50 \mu \mathrm{m}^{2}$ typical of conventional erbium doped fibres. The cavity is formed by the $4 \%$ Fresnel reflection at the pump launch end and the high reflector mirror, all other surfaces within the cavity being either anti-reflection coated or angle-polished. The Q-switch itself was an acousto-optic modulator which gave $85 \%$ coupling to the first-order dif-fracted beam under optimal focusing conditions. The laser was configured to run in the zero-order, Q-switch mode. An angle-tuned, dielectric bandpass filter of $1 \mathrm{~nm}$ width was included within the cavity to allow for laser tuning (between 1530 and $1560 \mathrm{~nm}$ ) and to further reduce the loss of energy to ASE. A polariser was also included in the cavity to ensure a (linearly) polarised output.

The laser was found to Q-switch stably for repetition rates between $200 \mathrm{~Hz}$ and $1.5 \mathrm{KHz}$. The upper limit was determined by our maximum pump power and the lower limit by the onset of pulse-pulse amplitude noise which proved difficult to suppress without compromise to the laser performance. The power characteristic for a number of operating conditions is shown in Fig. 2.

The slope efficiency for both $\mathrm{CW}$ and Q-switched operation is -0.36 with respect to incident power, and corresponds to approximately $70 \%$ quantum efficiency with respect to launched power illustrating that the fibre is still highly efficient despite its unconventional design. The laser output was characterised against pulse repetition frequency for the maximum launched power of $\sim 300 \mathrm{~mW}$ at an operating wavelength of $1558 \mathrm{~nm}$. In Fig. 3, we plot the output pulse energy against repetition rate, where it is seen that at the lowest repetition rates $(\sim 200 \mathrm{~Hz})$ for which stable pulsing is observed pulse energies as high as $180 \mu \mathrm{J}$ are obtained. A corresponding plot of both the pulse duration and pulse peak power is shown inset in Fig. 3. The pulse peak powers are derived by integrating the recorded pulse shape at each repetition rate and relating the pulse form to the measured pulse energy. 
It is seen in Fig. 3 that the pulse duration decreases with repetition frequency from $120 \mathrm{~ns}$ at $1 \mathrm{kHz}$ down to a duration of $45 \mathrm{~ns}$ at $200 \mathrm{~Hz}$ with a corresponding peak power of $3.6 \mathrm{~kW}$. This variation of peak power and duration is typical of a Q switch fibre laser. Note that slightly higher peak powers $(4.2 \mathrm{~kW})$ were obtained in our previously experiments by virtue of the fact we were using a shorter length of active fibre $(\sim 63 \mathrm{~cm})$ and were thus able to get shorter pulse durations ( $10 \mathrm{~ns})$ [7]. A plot of the $45 \mathrm{~ns}$ pulse form obtained at $200 \mathrm{~Hz}$ is shown inset in Fig. 2, the spectral width of the pulses in this instance was $0.15 \mathrm{~nm}$ and the spectrum was free from distortion due to the effects of Raman scattering: slightly narrower spectra $\sim 0.1 \mathrm{~nm}$ were obtained at the higher repetition rates.

Fig. 2 Laser power characteristics for a number of setups and operating conditions

All measurements made at operating wavelength of $1558 \mathrm{~nm}$

$\triangle \mathrm{CW}$ operation, with Q-switch, polariser and filter removed from cavity

Q-switch operation at $1 \mathrm{kHz}$

Q-switch operation at $200 \mathrm{~Hz}$

Inset: $3.6 \mathrm{~kW}, 45 \mathrm{~ns}$ pulse shape obtained at $200 \mathrm{~Hz}$ pulse repetition rate

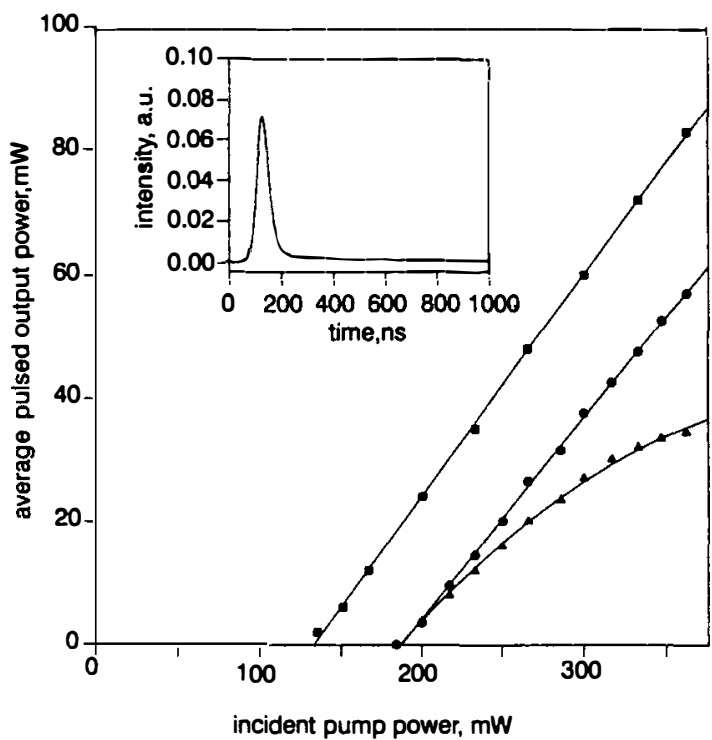

In conclusion, It is seen in Fig. 3 that the pulse duration decreases with repetition frequency from $120 \mathrm{~ns}$ at $1 \mathrm{kHz}$ down to a duration of $45 \mathrm{~ns}$ at $200 \mathrm{~Hz}$ with a corresponding peak power of $3.6 \mathrm{~kW}$. This variation of peak power and duration is typical of a $\mathrm{Q} \sim$ switch fibre laser. Note that slightly higher peak powers $(4.2 \mathrm{~kW})$ were obtained in our previously experiments by virtue of the fact we were using a shorter we have demonstrated an efficient all solid-state, Q-switch erbium doped fibre laser system based on large mode area erbium doped fibre. Pulse energies as high as $180 \mu \mathrm{J}$ have been obtained which, as far as we are aware, represent the highest pulse energies obtained to date from a single transverse mode fibre system. It would undoubtedly be possible to reduce the pulse duration down to the $<10 \mathrm{~ns}$ regime, with a resultant increase in peak power, simply by increasing the erbium concentration and thereby reducing the cavity length. Furthermore, significantly increased average powers $(>1 \mathrm{~W})$ and therefore correspondingly higher pulse repetition frequencies could be obtained by incorporating a large mode area inner core in a cladding pump fibre geometry [8].

Fig. 3 Pulse energy obtained against pulse repetition frequency.

Operating wavelength $1558 \mathrm{~nm}$

Inset: corresponding pulse duration and peak power against pulse repetition frequency

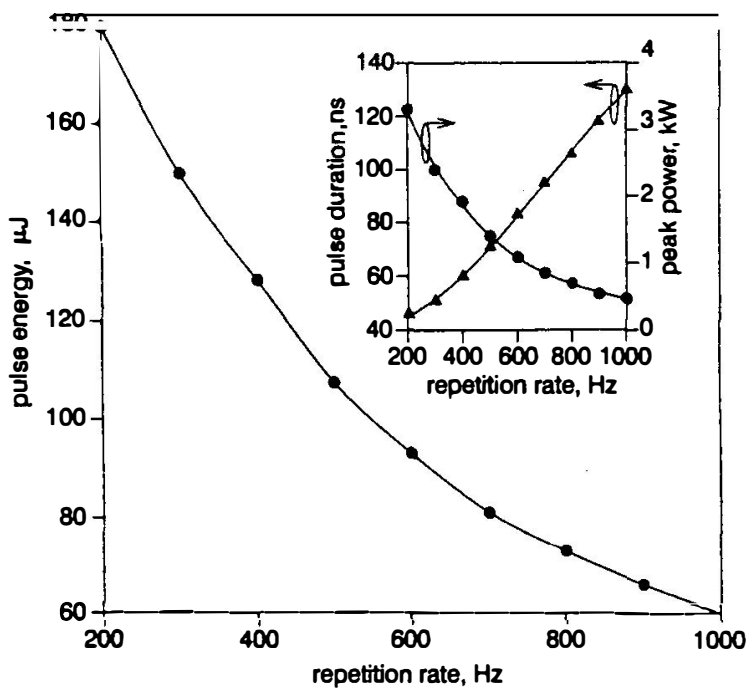

\section{References}

1 MEARS. R.J., REEKIE, L., POOLE. S.B., and PAYNE, D.N.: 'Low threshold tunable CW and Q-switched fibre laser operating at $1.55 \mu \mathrm{m}$ ', Electron. Lett., 1986, 22, pp. 159-160

2 SEGUIN, F., and OLESKEVICH. T.:'Diode-pumped Q-switched fibre laser', Opt. Eng., 1993, 32, (9), pp. 2036-2041

3 CHANDONNET, A., and LAROSE, G.: 'High power Q-Switch erbium fibre laser', Opt. Eng., 1993, 32, pp. 2031-2035

4 LEES, G.P., HARTOG, A., LEACH, A., and NEWSON, T.P.: '980nm diode pumped Er/Yb doped Q-switch fibre laser', Electron. Lett., 1995, 31, pp. $1836-1837$

5 NILSSON, J., and JASKORZYNSKA, B.: 'Modelling and optimisation of low repetition rate high energy pulse amplification in CW pumped erbium doped fibre amplifiers', Opt. Lett., 1993, 18, pp. 2099-2101

6 TAVERNER. D., RICHARDSON, D.J., DONG, L., CAPLEN, J.E., WILLIAMS, K., and PENTY, R.V.: ' $158 \mu 1$ pulses from a single transverse mode, large mode area EDFA', Opt. Leu., 1997, 22, pp. 37S-380

7 LEES, G.P., TAVERNER, D., RICHARDSON, D.J., DONG. L., and NEWSON. $\mathrm{A}$.P.: 'Q-switch erbium doped fibre laser using a large mode area fibre', Electron. Lett., 1997, 31, pp. 393-394

8 MINELLY. J.D., CHEN, Z.J., LAMING, R.I., and CAPLEN, J.C.: 'Efficient cladding pumping of an erbium fibre'. Proc. 21 st European Conf. Opt. Commun., Brussels, 1995, pp. 917-920 